\title{
Pneumonia and Extracorporeal Cardiopulmonary Resuscitation Followed by Targeted Temperature Management in Patients With Out-of-Hospital Cardiac Arrest
} - Retrospective Cohort Study -

\author{
Daiki Shiba, MD; Toru Hifumi, MD, PhD; Makiko Tsuchiya, MD; Kenji Hattori, MD; \\ Naoki Kawakami, MD; Kijong Shin, MD; Nozomi Fukazawa, MD; Katsuhiro Horie, MD; \\ Yu Watanabe, MD; Yohei Ishikawa, MD; Masato Shimizu, MD; Shutaro Isokawa, MD; \\ Nozomi Toya, MD; Tsutomu Iwasaki, MD; Norio Otani, MD; Shinichi Ishimatsu, MD, PhD
}

\begin{abstract}
Background: We examined the association between initiation of extracorporeal cardiopulmonary resuscitation (ECPR) and the incidence of infectious complications, such as pneumonia, sepsis, and bacteremia, after out-of-hospital cardiac arrest (OHCA) in patients who received targeted temperature management (TTM).

Methods and Results: This retrospective study used data from hospital medical records of patients with OHCA treated with TTM who had been admitted to St. Luke's International Hospital between April 2006 and December 2018. The primary endpoint was the association between the type of CPR and the incidence of early onset pneumonia in the intensive care unit (ICU; between $48 \mathrm{~h}$ and 7 days of hospitalization). Univariate and multivariate logistic regression analyses were performed for the primary endpoints. After applying the inclusion/exclusion criteria, 254 patients were included in the analyses; of these, 52 were enrolled in the ECPR group, and 202 were enrolled in the CCPR group. Median age was 58 years, $88.5 \%$ were male, prophylactic antibiotics were used in $80.3 \%$, and favorable neurological outcomes were observed in 51.9\%. On multivariate analysis, ECPR (odds ratio [OR], 2.78 ; $95 \% \mathrm{Cl}$ : 1.16-6.66; $P=0.037$ ) was significantly associated with the development of early onset pneumonia.
\end{abstract}

Conclusions: ECPR was an independent predictor of pneumonia after OHCA in patients who received TTM.

Key Words: Cardiopulmonary resuscitation; Extracorporeal circulation; Infection

$\mathbf{E}$ xtracorporeal cardiopulmonary resuscitation (ECPR) followed by targeted temperature management (TTM) has demonstrated significantly better outcome in patients with out-of-hospital cardiac arrest (OHCA). ${ }^{1-4}$ Despite this surprising outcome, however, critical complications, such as infection, hemorrhage, and ischemia, sometimes occur during ECPR management in the intensive care unit (ICU). ${ }^{5-7}$

Although a few observational studies did not note a significant association between infection complications and mortality in patients with OHCA or those managed with extracorporeal membrane oxygenation (ECMO), ${ }^{\mathbf{8}, 9}$ and several observational studies examining infection have been conducted in patients with ECPR, ,,5,10,11 the details of infectious complications during ECPR have not been fully examined. According to the Extracorporeal Life Support Organization (ELSO) general guidelines for all extracor- poreal life support (ECLS), there is no standard policy regarding prophylactic antibiotics for patients on ECLS, including ECPR, because of the lack of studies on infectious complications during ECPR. ${ }^{12}$ Therefore, we hypothesized that initiation of ECPR is a risk factor for infectious complications.

To address this hypothesis, this observational study examined the association between initiation of ECPR and the incidence of infectious complications, such as pneumonia, sepsis, and bacteremia, in patients with OHCA who received TTM, and also assessed infection management during ECPR.

Methods
Patients
This retrospective study used data from hospital medical
records of patients with OHCA treated with TTM who

Received August 23, 2019; revised manuscript received October 21, 2019; accepted October 25, 2019; J-STAGE Advance Publication released online November 20, 2019 Time for primary review: 1 day

Department of Emergency and Critical Care Medicine, St. Luke's International Hospital, Tokyo, Japan

The first two authors contributed equally to this work (D.S., T.H.).

Mailing address: Toru Hifumi, MD, PhD, Department of Emergency and Critical Care Medicine, St. Luke's International Hospital, 9-1 Akashi-cho, Chuo-ku, Tokyo 104-8560, Japan. E-mail: hifumitoru@gmail.com

ISSN-2434-0790 All rights are reserved to the Japanese Circulation Society. For permissions, please e-mail: cr@j-circ.or.jp 


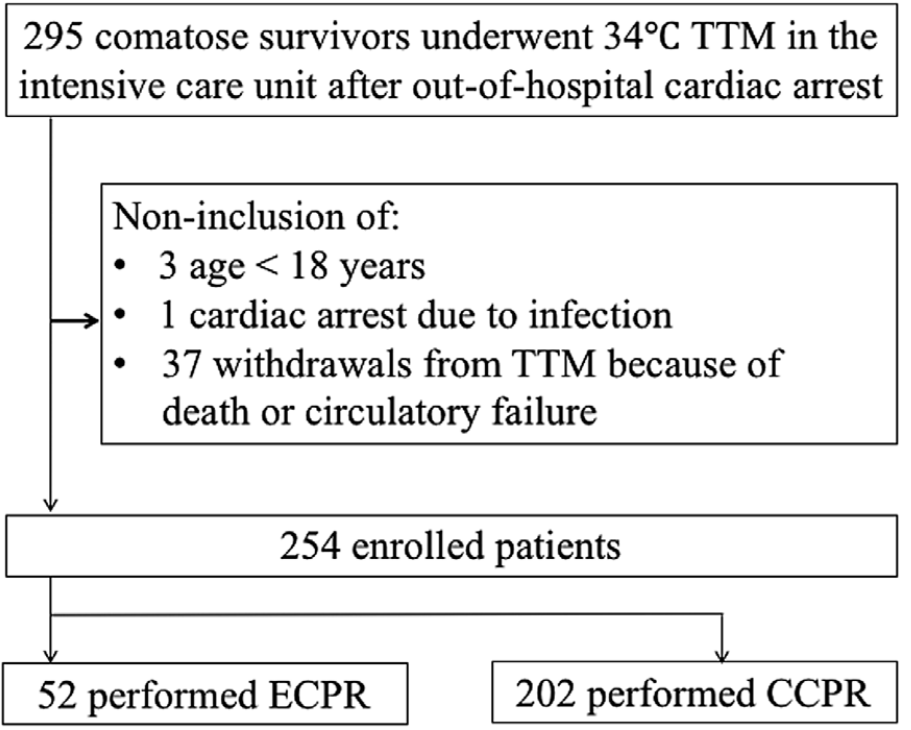

Figure 1. Patient selection. CCPR, conventional cardiopulmonary resuscitation; ECPR, extracorporeal cardiopulmonary resuscitation; TTM, targeted temperature management. had been admitted to the Critical Care Medicine unit of St. Luke's International Hospital between April 2006 and December 2018. Initial cardiac arrest (CA) rhythm was assessed by emergency medical service (EMS) personnel on arrival at the patient's location. Return of spontaneous circulation (ROSC) was defined as a return of palpable pulse in the carotid or radial artery, ${ }^{13}$ and its presence before hospital arrival was determined by EMS providers. The study was approved by the local ethics committee of St. Luke's International Hospital (approval number, 18-R099). Patients who received ECPR life support were assigned to the ECPR group and those who did not were assigned to the conventional CPR (CCPR) group. The exclusion criteria were age $<18$ years, withdrawal of therapeutic hypothermia (TH) because of death or circulatory failure, and infection or sepsis suspected as a cause of CA.

\section{ECMO and Post-Resuscitation Care}

The patients received standard CPR and post-CA care according to the 2005, 2010, and 2015 American Heart Association (AHA) guidelines. ${ }^{\mathbf{1 4}, 15}$ Before April 2013, the decision to initiate ECPR was made by physicians and cardiologists in the emergency department. From April 2013, patients with OHCA were eligible for ECPR mainly when the following criteria were met: (1) witnessed CA; (2) shockable initial rhythm; (3) non-response to conventional CPR; and (4) hospital arrival $\leq 30 \mathrm{~min}$ after $\mathrm{CA}$ and establishment of ECPR flow in $\leq 60 \mathrm{~min}$. ECMO was implanted by well-trained cardiologists and clinical engineers on a sterile field. Although inclusion criteria were established, the decision to initiate ECPR was made by the attending physicians in charge. Cannula of sizes 16.5 or 19.5 Fr were used for the femoral artery and those sized 19.5 or $21 \mathrm{Fr}$ were used for the femoral vein. The circuit for ECPR consisted of a heparin-bonded surface circuit, hollowmembrane oxygenator with an integral heat exchanger, and centrifugal blood pump (Capiox; TERUMO Corporation, Tokyo, Japan). The circuit was preorganized and primed with approximately $680 \mathrm{~mL}$ of saline. Once cannulation was achieved, 2,000 units of unfractionated heparin were given i.v., and the pump flow started initially at approximately $50 \mathrm{~mL} / \mathrm{kg} / \mathrm{min}$. For distal limb perfusion, anterograde reperfusion catheter to the distal artery was considered as necessary. Unfractionated heparin was infused to keep the activated clotting time to approximately 200-230s during ECMO

Patients underwent emergency coronary angiography, percutaneous coronary intervention, and placement of intra-aortic balloon pump (IABP) and pulmonary artery catheter (PAC) if necessary, and, subsequently, transport to the ICU. In the ICU, post-resuscitation care of both the ECPR group and CCPR group was initiated at the discretion of the attending physicians. Post-resuscitation care included oxygenation, adjustment of vasopressors, fluids, blood transfusion, and TH. TH was performed for OHCA patients who were unconscious after ROSC. All of the patients were mechanically ventilated, sedated (propofol/ fentanyl or midazolam/fentanyl depending on hemodynamic status) to maintain an appropriate sedation level, and paralyzed with muscle relaxants (rocuronium or vecuronium) during TTM. In the CCPR group, the core temperature was set as quickly as possible to $34^{\circ} \mathrm{C}$ with $4^{\circ} \mathrm{C}$ i.v. solutions and an external cooling device (Arctic Sun; C.R. Bard, NJ, USA), whereas in the ECPR group, the core temperature was managed by internal cooling. The target core temperature of $34^{\circ} \mathrm{C}$ was maintained for $24 \mathrm{~h}$, followed by gradual rewarming for the next $24 \mathrm{~h}$. Then, body temperature was maintained at normothermia until $72 \mathrm{~h}$ after ROSC. Transthoracic echocardiography was frequently performed to monitor left ventricular function and potential intraventricular thrombus formation. Weaning off ECMO was usually considered $48 \mathrm{~h}$ after initiation, when the patient was hemodynamically stable and adequately oxygenated. Withdrawal of ECMO was considered when there was irreversible multiple organ failure or severe neurological damage equivalent to brain death, but only after obtaining consent from the patient's relatives.

\section{Data Collection and Processing}

The following parameters were recorded for each subject: 


\begin{tabular}{|c|c|c|c|c|}
\hline Variables & $\begin{array}{c}\text { Total } \\
(n=254)\end{array}$ & $\begin{array}{l}\text { ECPR } \\
(n=52)\end{array}$ & $\begin{array}{c}\text { CCPR } \\
(n=202)\end{array}$ & P-value \\
\hline Age (years) & $58(48-67)$ & $54(41-62)$ & $58(49-68)$ & 0.024 \\
\hline Male sex & $225(88.5)$ & $49(94.2)$ & $176(87.1)$ & 0.220 \\
\hline Witnessed cardiac arrest & $223(87.8)$ & $50(96.2)$ & $173(85.6)$ & 0.054 \\
\hline Bystander CPR & $172(67.7)$ & $38(73.1)$ & $134(66.3)$ & 0.408 \\
\hline \multicolumn{5}{|l|}{ Initial rhythm } \\
\hline Vf & $201(79.1)$ & $42(80.7)$ & $159(78.7)$ & 0.476 \\
\hline VT & $1(0.4)$ & $0(0.0)$ & $1(0.5)$ & \\
\hline PEA & $34(13.4)$ & $9(17.3)$ & $25(12.4)$ & \\
\hline Asystole & $17(6.7)$ & $1(1.92)$ & $16(7.92)$ & \\
\hline Unknown & $1(0.4)$ & $0(0.0)$ & $1(0.5)$ & \\
\hline Shockable rhythm & $202(79.5)$ & $42(80.8)$ & $160(79.2)$ & 1 \\
\hline $\begin{array}{l}\text { Time from collapse to establishment of adequate } \\
\text { flow (min) }{ }^{\dagger}\end{array}$ & $\begin{array}{c}22 \\
(15.0-44.3)\end{array}$ & $\begin{array}{c}50 \\
(40.5-60.5)\end{array}$ & $\begin{array}{c}20 \\
(14.0-33.5)\end{array}$ & $<0.001$ \\
\hline \multicolumn{5}{|l|}{ Comorbidities } \\
\hline Hypertension & $94(37.0)$ & $18(34.6)$ & $76(37.6)$ & 0.749 \\
\hline Diabetes mellitus & $48(18.9)$ & $12(23.1)$ & $36(17.8)$ & 0.428 \\
\hline Hyperlipidemia & $39(15.4)$ & $6(11.5)$ & $33(16.3)$ & 0.519 \\
\hline Chronic kidney disease & $17(6.7)$ & $4(7.6)$ & $13(6.4)$ & 0.757 \\
\hline Heart failure & $16(6.3)$ & $5(9.6)$ & $11(5.4)$ & 0.333 \\
\hline Ischemic heart disease & $35(13.8)$ & $5(9.6)$ & $30(14.9)$ & 0.377 \\
\hline History of $\mathrm{PCl}$ & $17(6.7)$ & $3(5.7)$ & $14(6.9)$ & 1 \\
\hline History of CABG & $8(3.2)$ & $0(0.0)$ & $8(3.9)$ & 0.366 \\
\hline TIA, Stroke & $10(3.9)$ & $1(1.9)$ & $9(4.4)$ & 0.692 \\
\hline Asthma, COPD & $8(3.2)$ & $1(1.9)$ & $7(3.4)$ & 1 \\
\hline Malignancy & $9(3.5)$ & $2(3.8)$ & $7(3.4)$ & 1 \\
\hline No past medical history & $7(2.8)$ & $3(5.7)$ & $4(1.9)$ & 0.154 \\
\hline ECPR duration (days) & & $4(3-7)$ & $0(0-0)$ & 0.001 \\
\hline IABP support & $103(40.6)$ & $46(88.5)$ & $57(28.2)$ & 0.001 \\
\hline IABP duration (days) & $4(3-5)$ & $5(3.5-8)$ & $3(3-4)$ & 0.001 \\
\hline PAC & $70(27.6)$ & $47(90.4)$ & $23(11.4)$ & 0.001 \\
\hline PAC duration (days) & $5(4-7)$ & $5(4-8)$ & $4(3-6)$ & 0.001 \\
\hline SOFA score on ICU admission & $11(11-12)$ & $11(11-12)$ & $12(11-12)$ & 0.130 \\
\hline Prophylactic antibiotics use & $204(80.3)$ & $37(71.2)$ & $163(80.7)$ & 0.182 \\
\hline Ampicillin/sulbactam & $186(73.2)$ & $33(63.5)$ & $153(75.7)$ & 0.203 \\
\hline Others & $14(5.5)$ & $4(7.7)$ & $10(5.0)$ & \\
\hline None & $54(21.2)$ & $15(28.8)$ & $39(19.3)$ & \\
\hline ICU LOS (days) & $14(9.8-19)$ & $16.5(11-25.8)$ & $13(9-18)$ & 0.212 \\
\hline \multicolumn{5}{|l|}{ Outcome } \\
\hline CPC1 & $82(32.3)$ & $10(19.2)$ & $72(35.6)$ & 0.001 \\
\hline CPC2 & $50(19.7)$ & $8(15.4)$ & $42(20.8)$ & \\
\hline СРC3 & $53(20.8)$ & $8(15.4)$ & 45 (22.3) & \\
\hline CPC4 & $4(1.6)$ & $0(0.0)$ & $4(2.0)$ & \\
\hline CPC5 & $65(25.6)$ & $26(50.0)$ & $39(19.3)$ & \\
\hline Favorable neurological prognosis & $132(51.9)$ & $18(34.6)$ & $114(56.4)$ & 0.005 \\
\hline
\end{tabular}

Data given as median (IQR) or $\mathrm{n}(\%)$. ${ }^{+}$Time from collapse to ROSC in the CCPR group, or to ECMO in the ECPR group. CABG, coronary artery bypass grafting; CCPR, conventional cardiopulmonary resuscitation; COPD, chronic obstructive pulmonary disease; CPC, cerebral performance category; CPR, cardiopulmonary resuscitation; ECMO, extracorporeal membrane oxygenation; ECPR, extracorporeal cardiopulmonary resuscitation; IABP, intra-aortic balloon pumping; ICU, intensive care unit; LOS, length of stay; OHCA, out-of-hospital cardiac arrest; PAC, pulmonary artery catheter; $\mathrm{PCl}$, percutaneous coronary intervention; $\mathrm{PEA}$, pulseless electrical activity; ROSC, return of spontaneous circulation; SOFA, Sequential Organ Failure Assessment; TIA, transient ischemic attack; Vf, ventricular fibrillation; VT, ventricular tachycardia.

age; sex; presence of witnessed CA; presence of bystander CPR; initial rhythm; time from collapse to establishment of adequate flow (CCPR group, time from collapse to ROSC; ECPR group, time from arrest to ECMO); comorbidities; IABP and PAC insertion; prophylactic antibiotic use; ICU length of stay; and neurological outcomes. A favorable outcome was defined as cerebral performance category (CPC) 1 or $2,{ }^{16}$ whereas an unfavorable outcome was defined as CPC 3-5.

\section{Definitions}

Pneumonia Diagnosis of early onset pneumonia was 


\begin{tabular}{|lcccc|}
\hline \multicolumn{1}{|c|}{ Table 2. Incidence of Infectious Complications in OHCA Patients } & & \\
\multicolumn{1}{c}{ Variables } & $\begin{array}{c}\text { Total } \\
(\mathbf{n = 2 5 4 )}\end{array}$ & $\begin{array}{c}\text { ECPR } \\
(\mathbf{n = 5 2})\end{array}$ & $\begin{array}{c}\text { CCPR } \\
(\mathbf{n = 2 0 2})\end{array}$ & P-value \\
Pneumonia & $91(37.9)$ & $30(58.8)$ & $61(32.3)$ & 0.001 \\
Sepsis & $54(21.3)$ & $34(65.4)$ & $20(9.9)$ & 0.001 \\
\hline Bacteremia & $13(5.1)$ & $7(13.5)$ & $6(3.0)$ & 0.007 \\
\hline
\end{tabular}

Data given as $\mathrm{n}(\%)$. Abbreviations as in Table 1.

made on the basis of clinical signs and symptoms between $48 \mathrm{~h}$ and 7 days of hospitalization. The criteria were as follows: presence of new or progressive consolidation on chest X-ray together with at least 2 of 3 clinical features (i.e., fever $\geq 38.0^{\circ} \mathrm{C}$, leukocytosis or leukopenia $[>12,000$ cells $/ \mu \mathrm{L}$ or $<4,000$ cells $/ \mu \mathrm{L}$ ], purulent tracheobronchial sections)..$^{13,17}$

Sepsis Sepsis is defined as a life-threatening organ dysfunction caused by a dysregulated host response to infection. Organ dysfunction can be identified as an acute change in the total Sequential Organ Failure Assessment (SOFA) score ${ }^{14} \geq 2$ points consequent to the infection. The baseline SOFA score can be assumed to be zero in patients not known to have pre-existing organ dysfunction. ${ }^{15}$

Bacteremia Bacteremia is defined as at least 1 positive blood culture of a bacterium that is not part of the normal skin flora. Coagulase-negative staphylococci and Bacillus spp. were assessed separately to determine whether they were clinically significant pathogens or contaminants. The criteria of true bacteremia included multiple positive blood cultures or evidence of a primary site of infection with the same organism. ${ }^{18}$

\section{Endpoints}

The primary endpoint was the association between type of CPR (ECPR vs. CCPR) and the incidence of pneumonia diagnosed $\leq 7$ days after admission. The secondary endpoint was the association between type of CPR (ECPR vs. CCPR) and the incidence of sepsis and bacteremia during ICU stay.

\section{Statistical Analysis}

Baseline characteristics were compared using the Wilcoxon summed rank test for continuous variables and the chisquared test for binary and categorical variables as appropriate. Univariate and multivariate logistic regression analyses were performed for the primary endpoints. Multivariate analysis was adjusted for possible confounders: age $>65$ years; sex; presence of witnessed CA and bystander CPR; initial rhythm (shockable); time from collapse to establishment of adequate flow (time from collapse to ROSC in CCPR, or to ECMO in the ECPR group; ordinal variables based on quartiles because of the 2 different events [ROSC or ECMO] with apparently different median times [20 min in CCPR and $50 \mathrm{~min}$ in ECPR]), ECPR (vs. CCPR); diabetes as a comorbidity; SOFA score on admission; and initial prophylactic antibiotics. ${ }^{\text {19-22 }} \mathrm{We}$ performed a subgroup analysis involving patients who had received prophylactic antibiotics, particularly ampicillin/ sulbactam, and a shockable initial rhythm. Change in C-reactive protein (CRP) was compared between the ECPR group and CCPR group during the first 4 days after admission. Statistical analysis was performed using JMP version 11 (SAS Institute, Cary, NC, USA). Missing data were excluded on analysis.

\section{Ethics}

The study protocol and patient consent procedures were approved by the Institutional Review Board of St. Luke's International Hospital.

\section{Results}

Two hundred and ninety-five patients met the inclusion criteria. Of the total, 37 patients were excluded because of hemodynamic instability or death: 17 because of death in $\leq 24 \mathrm{~h}, 10$ because of death between 24 and $48 \mathrm{~h}$, and 10 due to cessation of TH because of circulatory failure. We also excluded another 3 patients $<18$ years old and 1 CA case because of infection. The remaining 254 patients were included in the analysis; of these, 52 were enrolled in the ECPR group, and 202 were enrolled in the CCPR group (Figure 1). Of the 254 patients, 7 had pulmonary embolism.

\section{Baseline Characteristics}

Overall, median patient age was 58 years, and $88.5 \%$ were male. ECPR was initiated in $20.4 \%$ of the patients, and prophylactic antibiotics were used in $80.3 \%$. Favorable neurological outcomes were observed in $51.9 \%$ of the patients. The baseline characteristics were compared between the ECPR and CCPR groups. There were significant differences in age, IABP support, duration of IABP use, PAC insertion, and duration of PAC use (Table 1).

Development of pneumonia, sepsis, and bacteremia was significantly more prevalent in the ECPR patients than in the CCPR patients $(58.8 \%$ vs. $32.3 \%, \mathrm{P}=0.001 ; 65.4 \%$ vs. $9.9 \%, \mathrm{P}<0.001$; and $13.5 \%$ vs. $3.0 \%, \mathrm{P}=0.007$, respectively; Table 2).

Details of the causes of bacteremia are given in Supplementary Table 1. Four cases of Staphylococcus epidermidis were observed in the ECPR group.

A comparison of baseline characteristics according to pneumonia status is shown in Supplementary Table 2. The median time from collapse to establishment of adequate flow was significantly longer in patients with pneumonia than in those without pneumonia (33.5 min; IQR, 17-48.8 min vs. $18 \mathrm{~min}, \mathrm{IQR}, 12-35 \mathrm{~min}, \mathrm{P}<0.001)$. The proportion of patients who received ECPR was significantly higher in the group that had pneumonia than in the group that did not $(32.9 \%$ vs. $13.5 \%, \mathrm{P}=0.001)$.

\section{ECPR and Infectious Complications}

Primary Analysis Regarding the primary endpoint, on multivariate ECPR (OR, 2.78; 95\% CI: 1.16-6.66; $\mathrm{P}=0.021)$ was significantly associated with the development of pneumonia (Table 3).

Secondary Analysis On multivariate logistic regression analysis to assess the association between ECPR and the development of sepsis and bacteremia, ECPR was significantly associated with sepsis (OR, 23.7; 95\% CI: 8.07-68.76; 
Table 3. Indicators of Pneumonia in OHCA Patients

\begin{tabular}{|c|c|c|c|c|}
\hline \multirow{2}{*}{ Variables } & \multicolumn{2}{|c|}{ Univariate analysis } & \multicolumn{2}{|c|}{ Multivariate analysis } \\
\hline & OR (95\% Cl) & P-value & OR $(95 \% \mathrm{Cl})$ & P-value \\
\hline ECPR & $2.99(1.58-5.65)$ & 0.001 & $2.78(1.16-6.66)$ & 0.021 \\
\hline Age $>65$ years & $1.57(0.89-2.76)$ & 0.118 & $1.65(0.85-3.21)$ & 0.135 \\
\hline Male sex & $1.25(0.53-2.91)$ & 0.602 & $1.23(0.48-3.13)$ & 0.662 \\
\hline Witnessed cardiac arrest & $0.61(0.28-1.34)$ & 0.22 & $0.26(0.09-0.74)$ & 0.013 \\
\hline Bystander CPR & $0.74(0.42-1.30)$ & 0.307 & $0.69(0.36-1.33)$ & 0.274 \\
\hline Shockable rhythm & $0.82(0.43-1.56)$ & 0.549 & $0.79(0.36-1.74)$ & 0.056 \\
\hline Diabetes mellitus & $0.69(0.34-1.38)$ & 0.294 & $0.62(0.28-1.38)$ & 0.248 \\
\hline SOFA score & $1.43(0.35-5.78)$ & 0.611 & $1.15(0.86-1.52)$ & 0.329 \\
\hline Prophylactic antibiotic use & $0.82(0.44-1.53)$ & 0.541 & $0.81(0.39-1.67)$ & 0.573 \\
\hline \multicolumn{5}{|c|}{$\begin{array}{l}\text { Time from collapse to establishment of adequate } \\
\text { flow (min) }{ }^{\dagger}\end{array}$} \\
\hline $0-15$ & Ref. & & Ref. & \\
\hline $16-22$ & $0.91(0.37-2.21)$ & 0.842 & $0.81(0.32-2.04)$ & 0.657 \\
\hline $23-44$ & $2.35(1.11-5.00)$ & 0.026 & $1.86(0.82-4.21)$ & 0.132 \\
\hline $44-110$ & $3.28(1.57-6.86)$ & 0.002 & $1.89(0.76-4.69)$ & 0.166 \\
\hline
\end{tabular}

tTime from collapse to ROSC in the CCPR group, or to ECMO in the ECPR group. Abbreviations as in Table 1.

Table 4. Multivariate Indicators of Pneumonia in OHCA Patients: Subgroup Analysis

\section{Models}

Prophylactic ampicillin/sulbactam use $(n=186)$

Shockable rhythm $(\mathrm{n}=202)$

\section{Variable}

ECPR
Multivariate analysis ${ }^{\dagger}$

$\begin{array}{cc}\text { OR (95\% Cl) } & \text { P-value } \\ 3.39(1.10-10.42) & 0.033 \\ 3.74(1.36-10.2) & 0.010\end{array}$

0.010

${ }^{\dagger}$ Adjusted factors were the same as those in the primary analysis: age, sex, witnessed cardiac arrest, bystander $\mathrm{CPR}$, initial rhythm (shockable), time from collapse to establishment of adequate flow, ECPR, diabetes mellitus, prophylactic antibiotic use. Abbreviations as in Table 1.

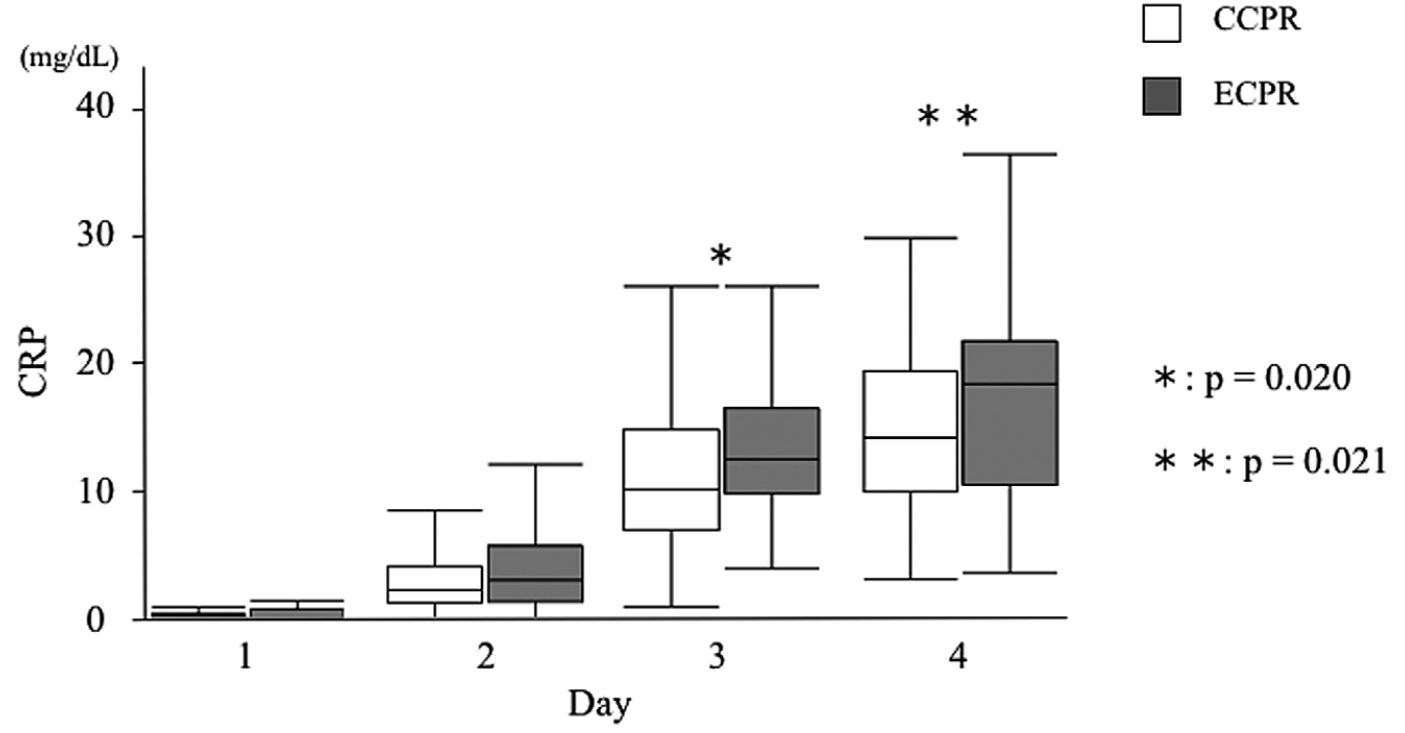

Figure 2. Change in C-reactive protein (CRP) during targeted temperature management in out-of-hospital cardiac arrest patients treated with conventional cardiopulmonary resuscitation (CCPR) or extracorporeal cardiopulmonary resuscitation (ECPR). 
$\mathrm{P}<0.001$; Supplementary Table 3) and bacteremia (OR, 7.56; 95\% CI: 1.41-40.8; P=0.018; Supplementary Table 4).

Subgroup Analysis During subgroup analysis, the significant association between ECPR and the development of pneumonia persisted in the patients who had received prophylactic antibiotics, particularly ampicillin/sulbactam, and who had shockable initial rhythm (Table 4). We chose these groups because early antibiotic use can affect the incidence of pneumonia, and shockable rhythm is one of the most important baseline characteristics.

\section{Change in CRP During TH}

There were no significant differences in CRP between the 2 groups on day 1 and day 2, but median CRP in the ECPR group progressively increased relative to that in the CCPR group, and significant differences were observed on days 3 and $4(12.3 \mathrm{mg} / \mathrm{dL}$; IQR, $9.6-16.4 \mathrm{mg} / \mathrm{dL}$ vs. $10.1 \mathrm{mg} / \mathrm{dL}, \mathrm{IQR}, 6.8-14.6 \mathrm{mg} / \mathrm{dL}, \mathrm{P}=0.020$; and $17.9 \mathrm{mg} / \mathrm{dL}$; IQR, 10.0-21.2 mg/dL vs. $13.6 \mathrm{mg} / \mathrm{dL}$; IQR, $9.5-18.8 \mathrm{mg} / \mathrm{dL}$, $\mathrm{P}=0.021$, respectively; Figure 2).

\section{Discussion}

In this observational study, initiation of ECPR was an independent predictor of infectious complications, that is, pneumonia, sepsis, and bacteremia, in patients with OHCA followed by TTM. Subgroup analysis also showed a strong association between ECPR and the development of pneumonia. CRP was significantly higher in the ECPR group than in the CCRP group on days 3 and 4 after admission.

\section{Comparisons}

The incidence of pneumonia after CA varies from $18 \%$ to $65 \%$ depending on the diagnosis criteria and patient populations. ${ }^{10,23}$ The incidence of pneumonia in patients who received ECPR in the current study was relatively high $(59.6 \%)$, but the incidence of pneumonia in the patients who did not receive ECMO (i.e., the CCPR group; 40\%) in the current study was similar to that in a previous study in which 765 OHCA patients were treated with TTM $(48 \%){ }^{24}$ Subsequently, we thought that an appropriate diagnosis could be established in the current study. An ELSO registry reported an incidence of pneumonia in OHCA patients treated with ECMO as only $12.9 \%,{ }^{5}$ but that study used a self-report system, and only $27.6 \%$ of the patients survived. Furthermore, the definition of pneumonia and number of patients treated with TTM were not described. Thus, a simple comparison could not be made because of the heterogeneity between the 2 studies.

\footnotetext{
Mechanism

In the present study, the ECPR group had a relatively longer time from collapse to establishment of adequate blood flow than the CCPR group (median, $50 \mathrm{~min}$ vs. $20 \mathrm{~min}$, respectively, $\mathrm{P}=0.001$ ). The longer low flow time was considered to be the reason for the development of pneumonia in the ECPR group. Low flow time is equal to the time of chest compression in the flat position. Infectious complications in the ECPR group can also be explained by the initial inflammatory response to extracorporeal circulation. ${ }^{25}$ The significantly higher level of CRP observed in the ECPR group in the early phase of admission indicates that ECPR was itself an inducer of inflammatory response. The initiation of ECPR is associated with an immediate and complex inflammatory reaction, such as the contact
}

system, coagulation, and complement cascade activation, similar to that seen in systemic inflammatory response syndrome. This innate immune response causes a persistent compensatory anti-inflammatory response, which may lead to immunoparalysis followed by infection. ${ }^{26,27}$

\section{Clinical Implementation}

Prophylactic antibiotics were used in $>70 \%$ of the patients who underwent ECPR, but pneumonia developed in approximately $60 \%$ of the ECPR group. Considering the fact that ampicillin/sulbactam was mainly used as the firstline antibiotics in the current study, therapies other than prophylactic antibiotics, such as hand hygiene, should be emphasized. Completion of ventilator-associated pneumonia (VAP) bundle may reduce the rate of infectious complications. Subgroup analysis also indicated that expected mild brain injury patients (who had an initial shockable rhythm) needed attention for infectious complications.

We emphasize that ECPR in itself does not harm OHCA patients, but that additional attention for infectious complications is required in ECPR care in the ICU. The development of infectious complications may not be directly associated with the outcome in OHCA patients who received TTM (Supplementary Table 5), but appropriate management for infection is one of the principal components of ECMO management.

\section{Study Limitations}

Several limitations of this study need to be addressed. First, details of VAP bundle completion were not examined because the dataset was unavailable. Second, the number of patients included in the study was moderate. Third, diagnostic criteria should be further discussed. Pulmonary contusion and edema can be diagnosed as pneumonia, and inflammatory response to ECMO and PCAS can be diagnosed as sepsis under the current definition. Fourth, the current data seem to be slightly outdated because standard CPR and post-CA care were performed according to the 2005 and 2010 AHA guidelines.

\section{Conclusions}

ECPR was an independent predictor of pneumonia in patients with OHCA who received TTM.

\section{Disclosures}

The authors declare no conflicts of interest.

\section{Author Contributions}

D.S. and T.H. were responsible for the conception, drafting, and revision of the manuscript. N.O. and S.I. helped to draft the manuscript. All authors read and approved the final manuscript and take full responsibility for all aspects of the study.

\section{References}

1. Wang HE, Schmicker RH, Daya MR, Stephens SW, Idris AH, Carlson JN, et al. Effect of a strategy of initial laryngeal tube insertion vs endotracheal intubation on 72-hour survival in adults with out-of-hospital cardiac arrest: A randomized clinical trial. JAMA 2018; 320: 769-778.

2. Maekawa K, Tanno K, Hase M, Mori K, Asai Y. Extracorporeal cardiopulmonary resuscitation for patients with out-of-hospital cardiac arrest of cardiac origin: A propensity-matched study and predictor analysis. Crit Care Med 2013; 41: 1186-1196.

3. Holmberg MJ, Geri G, Wiberg S, Guerguerian AM, Donnino MW, Nolan JP, et al. Extracorporeal cardiopulmonary resuscitation for cardiac arrest: A systematic review. Resuscitation 2018; 131: 
91-100.

4. Ortega-Deballon I, Hornby L, Shemie SD, Bhanji F, Guadagno E. Extracorporeal resuscitation for refractory out-of-hospital cardiac arrest in adults: A systematic review of international practices and outcomes. Resuscitation 2016; 101: 12-20.

5. Haas NL, Coute RA, Hsu CH, Cranford JA, Neumar RW. Descriptive analysis of extracorporeal cardiopulmonary resuscitation following out-of-hospital cardiac arrest: An ELSO registry study. Resuscitation 2017; 119: 56-62.

6. Otani T, Sawano H, Natsukawa T, Matsuoka R, Nakashima T, Takahagi M, et al. D-dimer predicts bleeding complication in out-of-hospital cardiac arrest resuscitated with ECMO. Am J Emerg Med 2018; 36: 1003-1008.

7. Beyea MM, Tillmann BW, Iansavichene AE, Randhawa VK, Van Aarsen K, Nagpal AD. Neurologic outcomes after extracorporeal membrane oxygenation assisted CPR for resuscitation of out-of-hospital cardiac arrest patients: A systematic review. Resuscitation 2018; 130: 146-158.

8. Sun HY, Ko WJ, Tsai PR, Sun CC, Chang YY, Lee CW, et al. Infections occurring during extracorporeal membrane oxygenation use in adult patients. $J$ Thorac Cardiovasc Surg 2010; 140: 1125 1132.e1122.

9. Mongardon N, Perbet S, Lemiale V, Dumas F, Poupet H, Charpentier J, et al. Infectious complications in out-of-hospital cardiac arrest patients in the therapeutic hypothermia era. Crit Care Med 2011; 39: 1359-1364.

10. Kagawa E, Inoue I, Kawagoe T, Ishihara M, Shimatani Y, Kurisu S, et al. Assessment of outcomes and differences between in- and out-of-hospital cardiac arrest patients treated with cardiopulmonary resuscitation using extracorporeal life support. Resuscitation 2010; 81: $968-973$.

11. Yannopoulos D, Bartos JA, Martin C, Raveendran G, Missov E, Conterato M, et al. Minnesota Resuscitation Consortium's advanced perfusion and reperfusion cardiac life support strategy for out-of-hospital refractory ventricular fibrillation. J Am Heart Assoc 2016; 5: e003732.

12. Extracorporeal Life Support Organization (ELSO). Guidelines for adult respiratory failure. https://www.elso.org/Portals/0/ ELSO $\% 20$ Guidelines $\% 20$ For $\% 20$ Adult $\% 20$ Respiratory $\% 20$ Failure\%201_4.pdf\#search=\%27Extracorporeal+Life+Support +Organization+General+Guidelines $\% 27$ (accessed November 11, 2019)

13. Johanson WG Jr, Pierce AK, Sanford JP, Thomas GD. Nosocomial respiratory infections with gram-negative bacilli: The significance of colonization of the respiratory tract. Ann Intern Med 1972; 77: 701-706.

14. Ferreira FL, Bota DP, Bross A, Melot C, Vincent JL. Serial evaluation of the SOFA score to predict outcome in critically ill patients. JAMA 2001; 286: 1754-1758.

15. Singer M, Deutschman CS, Seymour CW, Shankar-Hari M, Annane D, Bauer M, et al. The third international consensus definitions for sepsis and septic shock (Sepsis-3). JAMA 2016; 315: $801-810$.
16. Cummins RO, Chamberlain DA, Abramson NS, Allen M, Baskett PJ, Becker L, et al. Recommended guidelines for uniform reporting of data from out-of-hospital cardiac arrest: The Utstein Style. A statement for health professionals from a task force of the American Heart Association, the European Resuscitation Council, the Heart and Stroke Foundation of Canada, and the Australian Resuscitation Council. Circulation 1991; 84: 960-975.

17. Fabregas N, Ewig S, Torres A, El-Ebiary M, Ramirez J, de La Bellacasa JP, et al. Clinical diagnosis of ventilator associated pneumonia revisited: Comparative validation using immediate post-mortem lung biopsies. Thorax 1999; 54: 867-873.

18. Wiggers JB, Xiong W, Daneman N. Sending repeat cultures: Is there a role in the management of bacteremic episodes? (SCRIBE study). BMC Infect Dis 2016; 16: 286

19. Bernard SA, Gray TW, Buist MD, Jones BM, Silvester W, Gutteridge G, et al. Treatment of comatose survivors of out-ofhospital cardiac arrest with induced hypothermia. $N$ Engl J Med 2002; 346: $557-563$.

20. Hypothermia after Cardiac Arrest Study Group. Mild therapeutic hypothermia to improve the neurologic outcome after cardiac arrest. $N$ Engl J Med 2002; 346: 549-556.

21. Soga T, Nagao K, Sawano H, Yokoyama H, Tahara Y, Hase M, et al. Neurological benefit of therapeutic hypothermia following return of spontaneous circulation for out-of-hospital non-shockable cardiac arrest. Circ J 2012; 76: 2579-2585.

22. Inoue A, Hifumi T, Yonemoto N, Kuroda Y, Kawakita K, Sawano $\mathrm{H}$, et al. The impact of heart rate response during 48-hour rewarming phase of therapeutic hypothermia on neurologic outcomes in out-of-hospital cardiac arrest patients. Crit Care Med 2018; 46: e881-e888.

23. Perbet S, Mongardon N, Dumas F, Bruel C, Lemiale V, Mourvillier B, et al. Early-onset pneumonia after cardiac arrest: Characteristics, risk factors and influence on prognosis. $\mathrm{Am} \mathrm{J}$ Respir Crit Care Med 2011; 184: 1048-1054.

24. Nielsen N, Sunde K, Hovdenes J, Riker RR, Rubertsson S, Stammet $\mathrm{P}$, et al. Adverse events and their relation to mortality in out-of-hospital cardiac arrest patients treated with therapeutic hypothermia. Crit Care Med 2011; 39: 57-64.

25. Millar JE, Fanning JP, McDonald CI, McAuley DF, Fraser JF. The inflammatory response to extracorporeal membrane oxygenation (ECMO): A review of the pathophysiology. Crit Care 2016; 20: 387.

26. Adib-Conquy M, Cavaillon JM. Compensatory anti-inflammatory response syndrome. Thromb Haemost 2009; 101: 36-47.

27. Bone RC. Sir Isaac Newton, sepsis, SIRS, and CARS. Crit Care Med 1996; 24: 1125-1128.

\section{Supplementary Files}

Please find supplementary file(s);

http://dx.doi.org/10.1253/circrep.CR-19-0077 